International Journal of Child, Youth and Family Studies (2016) 7(3/4): 343-363

DOI: http://dx.doi.org/10.18357/ijcyfs73-4201616089

\title{
PREVALENCE OF INTIMATE PARTNER VIOLENCE IN JAMAICA: IMPLICATIONS FOR PREVENTION AND INTERVENTION
}

\section{Delores E. Smith}

\begin{abstract}
Intimate partner violence (IPV) is a global phenomenon. It is pervasive in every society and cuts across culture, religion, wealth, status, age, and lifestyle. IPV is a violation of women's human rights and a threat to public health and national development. However, in many societies, particularly developing nations, it is not given the national attention it deserves. The purpose of the current article is to present a snapshot of the prevalence and scope of IPV in the Jamaican context. In addition to presenting information on IPV and its consequences, the article uses ecological systems theory to delineate the various factors that potentially place Jamaican women at risk for intimate partner victimization. Further, the article proposes strategies for addressing existing cultural gender norms and beliefs about heterosexual interpersonal relationships and offers suggestions to policy makers for prevention and intervention approaches to limit the potential for the perpetration and maintenance of IPV. It is suggested that a combination of legislative action and public and private ventures will help reduce the incidence of domestic violence in Jamaica.
\end{abstract}

Keywords: Jamaica, domestic violence, intimate partner violence, gender, cultural norms

Delores E. Smith, PhD is Associate Professor in the Department of Child and Family Studies, College of Education, Health, and Human Sciences, 1215 W. Cumberland Ave., Knoxville, TN 37996. Email: delsmith@utk.edu 
International Journal of Child, Youth and Family Studies (2016) 7(3/4): 343-363

The United Nations defines intimate partner violence (IPV) as “a pattern of assaultive and coercive behaviours including physical, sexual and psychological attacks, as well as economic coercion used by adults or adolescents against their current or former intimate partners" (United Nations Children's Fund [UNICEF], 2006a, p. 3). It is one of the most ubiquitous human rights challenges of modern times (UNICEF, 2006a, 2006b; World Health Organization [WHO], 2005, 2013), pervading every culture, ethnicity, educational level, age, religion, income level, and lifestyle (UNICEF, 2006b; WHO, 2013). According to the World Health Organization (2013), IPV is a violation of women's human rights, a criminal justice issue, and a threat to public health and national development. The WHO deems IPV to be a peace and national security issue and hence urges that all societies make addressing violence against women a national priority.

Both men and women perpetrate IPV and are victimized by it. In fact, some authors (e.g., Dutton, 2007) have indicated that IPV perpetration is evenly distributed across gender, while others (e.g., Capaldi, Knoble, Shortt, \& Kim, 2012) have suggested that women commit IPV at higher rates than men. Nonetheless, the preponderance of the literature holds that women experience all forms of IPV at higher rates than men and that the consequences for women are more severe (Campbell, Glass, Sharps, Laughon, \& Bloom, 2007; Goussinsky \& Yassour-Borochowitz, 2012; Hart \& Klein, 2013; United Nations Development Programme [UNDP], 2012; WHO, 2013). According to the WHO (2016), an estimated 35\% of women are victims of IPV, and in some countries up to 71\% of women experience a lifetime occurrence of some form of IPV (WHO 2016). Furthermore, males kill their female partners at a rate 5 times higher than the reverse (Goussinsky \& Yassour-Borochowitz, 2012). According to the United Nations Office on Drugs and Crime (UNODC, 2014), globally, in 2012, almost half (47\%) of the 93,000 female victims of homicide were murdered by their intimate partner, whereas less than $6 \%$ of male homicide victims were murdered by an intimate partner. In the United States, a woman is nine times more likely to be murdered by her male partner than by a stranger (Campbell et al., 2007). Furthermore, 72\% of murder-suicides involved an intimate partner, with the woman murdered by her husband or boyfriend in 94\% of cases (Violence Policy Center, 2012). However, in Jamaica, as in many other developing societies, IPV has historically not been recorded separately from other types of violence but has been subsumed under the rubric of societal violence in general. Unfortunately, therefore, it has not received as much attention in the literature as have other forms of violence (e.g., political conflicts, community violence; Bott, Guedes, Godwin, \& Mendoza, 2012; UNDP, 2012).

\section{Purpose of the Paper}

The purpose of this article is to call attention to the prevalence and magnitude of IPV in Jamaica and to suggest approaches to addressing, and ultimately eliminating, the problem. In Jamaica, as in some other societies, a tradition of cultural acceptance of IPV makes it an especially contentious issue (Bott et al., 2012; Inter-American Commission on Human Rights [IACHR], 2012; Royes, Samiel, Tate, \& Fox, 2006). However, recent public discourse about its deleterious effects on Jamaican society has garnered increased civic interest in the issue and spurred some critics to charge that successive governments have not done enough to address it. According to some sources 
International Journal of Child, Youth and Family Studies (2016) 7(3/4): 343-363

(e.g., IACHR, 2012; Ustanny, 2006; Stop Violence Against Women, 2014), Jamaica lags behind many countries in taking action against violence against women. It has also been argued that a lack of committed responsiveness on the part of the state is the major cause of continued abuse of vulnerable women (IACHR, 2012; Stop Violence Against Women, 2014).

In this article, IPV is considered as a separate issue from the community violence in which it is embedded in many societies. Indeed, several sources (e.g., UNICEF, 2006a, 2006b; Stop Violence Against Women, 2014; WHO, 2005, 2013) have inferred that subsuming IPV under the rubric of broader societal violence renders official figures unreliable and the true magnitude of the problem difficult to decipher. That literature does not deny the robust interconnection between IPV and general community violence, but holds that the two are distinct. IPV is perpetrated mostly by men against women, primarily within the confines of intimate relationships and in the privacy of the home "behind closed doors" (WHO, 2013). Community violence, on the other hand, is committed in public areas, and usually by a perpetrator who is not in an intimate relationship with the victim (National Child Traumatic Stress Network, 2015). Furthermore, special recognition must be paid to IPV because of its destructive impact on society's most basic institution - the family, and most vulnerable members - women and children (Bott et al., 2012; UNICEF, 2006a, 2006b; WHO, 2013). As well, over the past three decades a strong body of research, albeit primarily from developed societies, has offered new knowledge about the nature of IPV and its detrimental social consequences.

Limited attention has been paid to IPV in the Jamaican setting (Stop Violence Against Women, 2014; Ustanny, 2006), and the major public health problems it presents for personal and national development (WHO, 2005, 2013, 2016). While not disputing that IPV also has male victims, the emphasis here is exclusively on female victims in the Jamaican context. This article is not meant to be an exhaustive review of IPV: it addresses only related factors that have been shown to be consistent across cultures. I review the literature on the prevalence of IPV in the Jamaican context, outline my theoretical framework, summarize the literature on the consequences of IPV, and note their implications for Jamaican society. I also make suggestions to policy makers and the private sector regarding workable strategies for addressing the ingrained cultural dynamics of IPV.

\section{The Jamaican Context}

Jamaica is a small island nation (4421 sq mi) with a population of 2.9 million. Women make up about $50 \%$ of the adult population. Within the UN economic and geographical classification, the island is an upper-middle-income economy situated within the Latin American and Caribbean (LAC) region. The LAC region is ranked among the most violent in the world , and Jamaica has the world's sixth-highest homicide rate (UNODC, 2014). Although there is no comprehensive prevalence data on IPV in Jamaica (Organization for Economic Co-operation and Development [OECD], 2014), IPV has been identified as the most prevalent form of violence on the island (Bott et al., 2012); however, for the most part, addressing the problem is not prominent in the country's national security blueprint (IACHR, 2012; Stop Violence Against Women, 2014). According to the Organization of American States (IACHR, 2012), IPV is one of the more common forms of 
International Journal of Child, Youth and Family Studies (2016) 7(3/4): 343-363

violence plaguing Jamaican society: "It contributes to the overall pattern of crime and violence due to its debilitating effects on the social fabric and its role in socializing the youths to violence as a means of dispute resolution” (p. 76). The IACHR (2012) also noted that, notwithstanding the government's efforts to adopt a series of international legal standards regarding gender equality and violence against women, Jamaican women continue to suffer discrimination and violence of all kinds, including all forms of IPV and sexual harassment.

Violence against women permeates all social strata of Jamaican society and, as is also true globally, its occurrence is significantly underreported due to prevailing social and cultural norms (OECD, 2014; IACHR, 2012; UNICEF, 2006b). In particular, because IPV often goes unreported, the number of incidents is much higher than recorded and presented in official reports (IACHR, 2012). However, the limited available data have indicated that of the 22,739 cases of domestic violence reported over a 3-year period (2005-2008), 61\% of the victims were women and girls (IACHR, 2012). Police statistics show that in 2013, there were 814 cases of women being raped and 128 women were murdered, 76 in what were classified as “domestic related” murders; also, of 52 cases of suicide in that year, $19.2 \%$ were attributed to domestic conflict (presumably an underestimate, as the motive in $45 \%$ of the cases was stated to be unknown; Planning Institute of Jamaica, 2014). One study (Le Franc, Samms-Vaughan, Hambleton, Fox, \& Brown, 2008) investigating the prevalence of interpersonal violence in three Caribbean countries found that Jamaican women reported the highest rate (85.1\%) of violent victimization within their intimate relationships; $45.3 \%$ of women in the Jamaican sample reported that they had experienced physical violence, and $72.6 \%$ indicated that they had been sexually coerced. In one study (Royes et al., 2006) utilizing focus groups, all the men from a middle- or upper-class background, and 80\% of other men, admitted to physically abusing an intimate partner. Findings from Soyibo and Lee's (2000) study indicated that $45 \%$ of their school-aged sample reported that they had witnessed IPV in their homes; 39\% had witnessed family members being injured in those violent encounters; and 24\% indicated that they had been injured themselves in violent domestic conflicts. In a report of IPV studies (UNICEF, 2006b), 50\% of Jamaican men acknowledged having hit their partner and 30\% of adolescents worried about the violence in their home. In the 2008 Reproductive Health Survey (RHS), Serbanescu, Ruiz, and Suchdev (2010) found that 35\% of Jamaican women (aged 15-49) reported a lifetime occurrence of at least one form of IPV (verbal, physical, or sexual); and 17\% reported having been subjected to any form of IPV over the past 12 months.

\section{Theoretical Framework of IPV}

Traditional theories seeking to explain male violence against women have used three lenses: biological, psychological, and sociological. According to Cunningham et al. (1998), there are conceptual variations among these broad frameworks: (a) biological explanations of male violence against women posit either a gene-based evolutionary process resulting in the male's instinctual struggle for dominance, or individual physiological factors such as brain injury; (b) psychological explanations emphasize the role of a disordered psyche; and (c) sociological explanations underscore the role of the social structures and systems that create and perpetuate male violence 
against women. However, contemporary thought maintains that because no single perspective can explain human outcomes, violence is best viewed through a set of integrative formulations (WHO, 2010, 2013). Hence, ecological systems theory, proposed by Bronfenbrenner (1979), has been identified as the best suited to address IPV. The WHO regards it as the gold standard for organizing and integrating the various nested mechanisms of human development (WHO, 2010, 2013). According to the ecological systems framework, human development occurs within a complex web of social exchanges among individuals and the multiple social systems in which they reside. Thus, the theory implies the importance of understanding human behaviour within the context of the interaction of numerous factors of the cultural ecology. In conceptualizing and studying IPV, therefore, the use of ecological systems theory is recommended because of the multiple interconnected risks that contribute to its perpetration and maintenance (WHO, 2010).

The model suggests four levels of inquiry: individual, relationship, community, and society. At the individual level, the model addresses the biological characteristics and personal characteristics and histories that engender IPV. Variables at this level include gender, history of violence, excessive alcohol use, education, and wealth status. At the relationship level, the social alliances that encourage or support the perpetration or victimization of IPV are considered. Factors such as economic inequality, excessive controlling behaviours, and isolation from potential social support are implicated. Major contributors at the community level include the neighbourhood climate, community policing and law enforcement practices, economic opportunities, and access to services. Lastly, the broader societal sphere encompasses factors such as cultural norms and national policies and laws - or lack thereof - that promote social inequalities among groups within the society (Gass, Stein, Williams, \& Seedat, 2011; WHO, 2010, 2013). For the sake of parsimony, in this article the four levels are collapsed into two: the proximal, comprising the individual and relational contexts; and the distal, comprising the community and cultural environments.

\section{Proximal Factors}

Many of the proximal risk factors regarded as key contributors to the occurrence and persistence of IPV in Jamaica are comparable to those seen in other cultures. For example, international research has shown a strong correlation between IPV and childhood exposure to violence (Bott et al., 2012; Gass et al., 2011; Hart \& Klein, 2013; UNICEF, 2006a). Consistent with those findings, data from the 2008 RHS (Serbanescu et al., 2010) indicated that the three strongest predictors of IPV victimization among Jamaican women were a history of childhood exposure to family violence, controlling behaviours of the intimate partner, and alcohol use. Other relevant factors were age, education, and wealth status.

\section{Childhood History of Violence}

Globally, having personally experienced or witnessed violence in the family of origin is the most robust predictor of IPV perpetration and victimization. Rates of IPV victimization are reportedly higher among women whose husbands were abused as children or who witnessed their 
mothers being abused (Bott et al., 2012; Hart \& Klein, 2013; WHO, 2010). Middlebrooks and Audage (2008) reported that children who witnessed IPV were four times more likely to report IPV perpetration in adulthood than children who did not witness IPV. These findings were confirmed by Gass et al. (2011), who found that men who reported IPV perpetration were four times as likely to have experienced childhood familial abuse and to have witnessed interparental violence. Having been battered as a child significantly increases the risk for both victimization and perpetration of family violence in later life (Bott et al., 2012; Capaldi et al., 2012; Gass et al., 2011). The literature also consistently demonstrates a strong overlap between children witnessing IPV and being abused or neglected (Hart \& Klein, 2013; Osofsky, 2003). Children who witnessed IPV were 15 times more likely than their non-witnessing peers to be physically abused (Osofsky, 2003).

In light of the strong correlations between childhood histories of violence and the potential for IPV, it is significant that harsh physical punishment features prominently in Jamaican childrearing practices. According to data from the 2011 Multiple Indicator Cluster Survey (MICS, Statistical Institute of Jamaica \& United Nations Children's Fund [STATIN-UNICEF], 2013), 85\% of Jamaican children aged 2 to 14 experienced some form of violent discipline at the hands of a parent or primary caregiver in the month prior to data collection. Gender and wealth status affected the risk of such treatment, in that $90 \%$ of children from the poorest quintile experienced violent discipline compared to $76 \%$ of children from the richest quintile, and boys received more frequent and severe punishment than did girls. For example, "Male children were more likely to be subjected to both 'any physical punishment' and 'severe physical punishment' (71.4 and 6.7\%) than female children (65.2 and 4.7\%)” (STATIN-UNICEF, 2013, p.10). A comparison of RHS data among 11 LAC countries indicated that Jamaican respondents registered the highest percentage (69.5) of women who experienced childhood physical violence by a family member (Bott et al., 2012). The overwhelming majority (82\%) of IPV victims in the 2008 Jamaica RHS reported that they had experienced childhood physical abuse by their parents, and a sizable minority (32\%) had witnessed interparental violence prior to age 15 (Serbanescu et al., 2010). Bott and associates (2012) observed that the prevalence of IPV victimization in Jamaica was twice as high among women who reported seeing their mothers being beaten than those who had indicated not witnessing such violence. Furthermore, women who had experienced IPV were significantly more likely than the control group (64.5\% vs. 52.1\%) to report the use of corporal punishment as a disciplinary measure on their own children (Bott et al., 2012).

\section{Controlling Behaviours}

Controlling behaviours are a form of IPV that perpetrators use to maintain dominance over their victims (Hart \& Klein, 2013; WHO, 2005). Men who exhibit controlling behaviours in their intimate relationships tend to also engage in other forms of IPV (Bott et al., 2012; Hart \& Klein, 2013; WHO, 2005); even on their own, such behaviours may be as emotionally injurious to victims as physical or sexual violence (WHO, 2005; Hart \& Klein, 2013). Across the LAC region, women who reported being subjected to three or more controlling behaviours by their intimate partners were up to three times more likely to report other forms of IPV compared to a control group (Bott et al., 2012). Similarly, the Jamaica RHS data showed that $75 \%$ of Jamaican women who experienced 
International Journal of Child, Youth and Family Studies (2016) 7(3/4): 343-363

physical or sexual IPV had experienced at least one controlling behaviour from their intimate partner, and having experienced multiple controlling behaviours was strongly associated with all forms of IPV (Serbanescu et al., 2010). The Jamaica RHS also indicated that the most frequently cited forms of controlling behaviours reported by participating women were: male partners tracking female partners (35\%), males being angry if their female partners spoke to another man (30\%), and males often being suspicious that female partners were unfaithful (20\%). Similar findings regarding the prevalence of controlling behaviours have been reported globally (Hart \& Klein, 2013; Laisser, Nyström, Lugina, \& Emmelin, 2011).

\section{Alcohol Use}

Across the globe, research findings have consistently implicated alcohol use in the perpetration of IPV. There is no evidence of a causal link between alcohol and IPV, but the majority of pertinent research has suggested a linear association between a perpetrator's alcohol use and the severity and frequency of partner violence (Hart \& Klein, 2013; Wilson, Graham, \& Taft, 2014). Specifically, men who misuse alcohol are up to 5 times more likely to perpetrate IPV against their intimate partners (WHO, 2010). Additionally, Gass et al. (2011) found that alcohol abuse was a strong predictor of IPV perpetration and victimization for both men and women. Male perpetrators were twice as likely as non-perpetrators to report alcohol abuse and female perpetrators were seven times as likely to have had alcohol use problems. Moreover, alcohol use has been associated with the severity of all forms of intimate partner abuse in several studies (Hart \& Klein, 2013).

Nevertheless, the ways in which alcohol contributes to IPV are complex. Some sources (e.g., Hart \& Klein, 2013; Wilson et al., 2014) have hypothesized that alcohol facilitates violence in various ways. It may help to break down the abuser's inhibitions, warp information processing and problem-solving abilities, increase risk-taking behaviours, and cause perpetrators to misinterpret their victims' comportment toward them (Wilson et al., 2014). Alcohol may also elevate the user's sense of personal power and dominance over others, thereby enabling and escalating the severity of the abusive behaviour (Hart \& Klein, 2013). Across the LAC region, "drunkenness or drug abuse” by a partner was the single most-commonly cited trigger for IPV (Bott et al., 2012). In the Jamaica RHS, nearly one-fourth (22\%) of abused women, compared to $11.2 \%$ of non-abused, reported that their intimate partner got drunk at least once within the past year (Serbanescu et al., 2010). Across the LAC region, both IPV victims and perpetrators "were more likely to be drinkers of alcohol (versus abstainers) and - among drinkers - more likely to drink heavily on each occasion, compared with those who did not report intimate partner violence” (Bott et al., 2012, p.111). Findings from several cross-sectional studies from lower- and middle-income countries have indicated that men who misuse alcohol are between 2 and 5 times more likely than non-consumers to perpetrate IPV (WHO, 2010).

\section{Socioeconomic Status}

The term socioeconomic status (SES) comprises education, income, and occupation. It is commonly used to conceptualize the social standing of an individual or group; in general, individuals with higher education tend to have higher incomes and more prestigious occupations 
International Journal of Child, Youth and Family Studies (2016) 7(3/4): 343-363

and hence, higher social status (Hart \& Klein, 2013; Serbanescu et al., 2010). IPV pervades all social classes, but low SES elevates its risk and severity (Benson \& Fox, 2004; WHO, 2010). Compared to their more affluent counterparts, women at the lower end of the socioeconomic scale are disproportionately victimized by their intimate other (Benson \& Fox, 2004; Hart \& Klein, 2013; WHO, 2010). The odds of a man battering his spouse increase significantly among poor, unemployed, and marginalized men (Benson \& Fox, 2004; Capaldi et al., 2012). For instance, Benson and Fox (2004) found that couples experiencing high financial strain were 3 times more likely to report IPV than couples with a low financial burden. In one study (Ackerson, Kawachi, Barbeau, \& Subramanian, 2008), women with no formal education were almost 6 times (5.51) as likely to experience IPV victimization as college-educated women, and wives of uneducated men were almost twice (1.84) as likely to be victimized by their partners than were wives of collegeeducated men.

Consistent with that literature, in the Jamaica RHS, lower educational attainment and concomitantly lower wealth status was associated with higher prevalence of IPV (Serbanescu et al., 2010). Women reporting the highest prevalence of lifetime IPV victimization had the lowest educational attainment and wealth status. Also, women with lower educational attainment (less than 9 years) were significantly more likely than those with higher education (more than 13 years) to subscribe to traditional gender roles, such as agreeing that a man should show his partner who is boss, and that a woman should obey her partner even when she disagrees (Serbanescu et al., 2010). The women least likely to disclose the abuse were of lower educational level (less than 9 years) and the poorest wealth status (i.e., poorest quintile; Serbanescu et al., 2010).

\section{Age}

IPV occurs across all stages of the lifespan. However, older age is associated with decreased risk for IPV, with the greatest risk occurring in late adolescence and young adulthood (Capaldi et al., 2012). Globally, women aged 16 to 24 are more likely than those in older age groups to be victimized by an intimate partner, and younger men are more likely than older men to perpetrate IPV (Johnson \& Das, 2009; UNICEF, 2006a; WHO, 2010). Consistent with that literature, younger Jamaican women (15-24 years) reported higher levels of IPV within the preceding 12 months than those in older cohorts (Bott et al., 2012; Serbanescu et al., 2010; STATIN-UNICEF, 2013). The younger cohort was also more likely to believe that a husband is justified in beating his wife for at least one specified reason (Serbanescu et al., 2010). Perhaps not surprisingly, the women least likely to disclose abuse were from the younger cohort (15-24 years; Serbanescu et al., 2010).

\section{Acceptance of Violence}

Across cultures, subscribing to the notion that violence is acceptable is strongly correlated with higher rates of IPV (Johnson \& Das, 2009; WHO, 2010). For example, embracing wife-beating as acceptable was found to increase the incidence of IPV fourfold in Bangladesh (Johnson \& Das, 2009). In one Jamaican study, employing focus groups and survey methodology, Royes and colleagues (2006) found that although violence against women was outwardly condemned, it was still condoned under certain conditions by both sexes. For example, in one focus group, while the 
International Journal of Child, Youth and Family Studies (2016) 7(3/4): 343-363

majority of the men agreed that was improper to hit a woman, some stated "there were instances when women 'frustrate' men into violent behavior" and that women required "a few blows now and then to show them who is boss” (p.46). It is also noteworthy that some women believed that there are times when women provoked the abuse and thus deserved to be beaten, and there was also general agreement among the women that "a man should be the head of his home and be the dominant figure in the relationships” (p. 46).

Researchers (e.g., Laisser et al., 2011) have maintained that over time social norms become internalized, leading women to believe that a husband has the right beat his wife. In a multination study of women's health, women's acceptance of wife-beating was higher among women who had experienced abuse than among the control group (WHO, 2005). Perhaps, then, repeated and extended exposure to abuse leads some women to accept violence against them as normal. It might be that “... women learn to 'accept' violence in circumstances where they themselves are victims, or that women who see violence as 'normal' are more likely to enter or remain in violent relationships” (WHO, 2005, p. 20). Research is lacking on the dynamics relating to IPV in Jamaica. However, it is likely that the entrenched belief that male violence against women is a matter of "men being men" contributes to women's acceptance and tolerance of violence against them by their intimate partners (Ustanny, 2006).

\section{Geography}

It has been widely shown that rural women are more likely to be victims of IPV than their urban or suburban counterparts. Rural women experience both higher rates and greater severity of IPV than their non-rural peers (Hart \& Klein, 2013; Peek-Asa et al., 2011; Zakar, Zakar, \& Abbas, 2016). Although the mechanisms linking geography and IPV are unclear, several factors peculiar to the rural setting have been hypothesized. For example, compared to their non-rural counterparts, rural women tend to have lower education and income, have stronger belief in and greater acceptance of patriarchal norms, marry at a younger age, and experience greater social isolation; all of these factors place these women at higher risk for IPV (Ackerson et al., 2008; Peek-Asa et al., 2011; WHO, 2010; Zakar et al., 2016). In fact, research has shown that rural women are 3 times further away than urban women from IPV intervention services such as emergency shelters, counselling, legal representation, transportation, and resources referral (Peek-Asa et al., 2011). They also have less access to information from the media or the internet (Hart \& Klein, 2013). Furthermore, rural women have fewer capacity-building opportunities and less access to economic resources, and so are more dependent on their intimate partners for their day-to-day survival (Zakar et al., 2016). In addition, because of rural women's lower educational levels and weaker social position, their awareness of their social and legal rights is severely compromised (Peek-Asa et al., 2011; Zakar et al., 2016). In one report of IPV in Jamaica (Serbanescu et al., 2010), geography was associated with women's experience of IPV. For example, rural women were more likely to subscribe to traditional gender roles and report higher rates of controlling behaviours by their intimate partner than their non-rural peers. Compared to their non-rural peers, rural women more frequently agreed that it is important that a man shows his partner who is boss, that a "good" wife obeys her husband, and that family problems be discussed only within the family. Furthermore, 
International Journal of Child, Youth and Family Studies (2016) 7(3/4): 343-363

women reporting four or more controlling behaviours by an intimate partner were more likely to reside in rural parishes. However, contrary to findings from some international studies (e.g., PeekAsa et al., 2011; Zakar et al., 2016), although there was a trend to rural Jamaican women reporting being recipients of verbal abuse at slightly higher rates than their peers, their reports of physical and sexual abuse did not differ significantly from non-rural women. In another study (STATINUNICEF, 2013), rural Jamaican women were also more likely to agree that men were justified in battering their partners.

\section{Distal Environment}

In terms of ecological systems theory, distal processes are those that have an indirect or secondary influence on development and behaviour. They include features of the sphere outside the home that still affect the interactions within the familial milieu. Social conditions that facilitate or impede optimal outcomes are part of the distal environment, and include neighbourhood and societal attributes that foster violence.

\section{Neighbourhood Quality}

The social conditions of a community affect the interactions of the people residing within it. For example, high levels of economic and social deprivation, unemployment, and crime and violence intensify the prevalence and severity of dysfunctional behaviours (Benson \& Fox, 2004; Edwards, Mattingly, Dixon, \& Banyard, 2014). Benson and Fox (2004) investigated the effects of individual and community risks and found that the highest rates of IPV were among women residing in neighbourhoods with a high incidence of individual poverty, unemployment, and job instability. In Benson and Fox's study, women whose intimate partners had a history of unstable employment stood a threefold risk of IPV victimization compared to those in the control group. Benson and Fox, therefore, reasoned that individual poverty combined with social deprivation creates a volatile mix that elevates a woman's risk of IPV. Those findings are in line with social organization theory, which posits that neighbourhood disadvantage increases the likelihood of "bad behaviours” among its residents and raises the probability of crime and disorder. For example, high criminal activity, and by extension lawlessness, within a community limits collective efficacy, impedes the community's ability to exert formal and informal social controls (Edwards et al., 2014), and increases women's vulnerability to violence, particularly rape (Ustanny, 2006).

In terms of the Jamaican ecology, high crime levels are a constant feature of Jamaican life, particularly in the poor urban communities; violence against women is therefore likely to be consistently higher in those locations (Ustanny, 2006) than in more privileged urban communities. In impoverished neighbourhoods, there is a high rate of vigilante violence and gang violence fed by the illegal gun trade; gangs exercise social control through violence and intimidation (Freedom House, 2015; IACHR, 2012). In one urban Jamaican study (Meeks-Gardner, Powell, Thomas, \& Millard, 2003), 1,710 youth aged 9 to 17 were asked about their perceptions of their neighbourhoods. Only 28\% of youth thought their neighbourhoods were very safe; $54 \%$ witnessed relationship fights between men and women; $60 \%$ had a family member who had been a victim of 
International Journal of Child, Youth and Family Studies (2016) 7(3/4): 343-363

violence, and 37\% had a family member who had been killed. Remarkably, the overwhelming majority (84\%) knew of peers who carried weapons to school, and the most common reasons for carrying a weapon included threatening or hurting others (66\%), for protection (64\%), or to gain respect (50\%). These dynamics have led observers (IACHR, 2012) to underscore the widespread insecurity, fear, and despair among the Jamaican populace. Furthermore, others (e.g., Smith, 2016; UNICEF, 2006b; Ustanny, 2006) have asserted that the cumulative effect of the many forms of violence (i.e., home, school, community, and media) to which Jamaican young people are exposed, have led to their desensitization to violence and the belief that violence of any kind and in any relationship is acceptable and normal. The Jamaica RHS (Serbanescu et al., 2010) did not disaggregate the IPV data by neighbourhood, but Kingston, the capital city of the island, is one of the three parishes with the highest percentage (30\%) of women reporting having experienced any physical or sexual IPV. It is also instructive that in the same study, male residents of the Kingston metropolitan area reported the highest level of agreement with statements affirming that a man was justified in beating his wife under certain conditions such as infidelity (27.3\%) and disobeying her husband (25\%).

\section{Social and Cultural Gender Norms}

A cultural acceptance of women's subordination normalizes violence against women and contributes to the perpetuation of spousal abuse (WHO, 2013). In Jamaica, widespread violence against women has been engendered by deep-seated patriarchal beliefs: the male is superior to the woman, the woman belongs to the man (Ustanny, 2006), the man is divinely sanctioned as the head of the household and gatekeeper of family resources, and it is the man's duty to keep the woman in her proverbial place (Stop Violence Against Women, 2014; Ustanny, 2006). It is contended that such traditional beliefs and attitudes have bolstered the acceptance of IPV in Jamaica and allowed offenders to commit acts of violence against women with impunity (Bott et al., 2012; IACHR, 2012; Royes et al., 2006; Stop Violence Against Women, 2014). Further, Ustanny (2006) argued that IPV in Jamaica is due, among other factors, to the compounding effect of numerous deeprooted cultural attributes including gender socialization that teaches women to be subservient and dependent on men. She also criticized the local media for seemingly not taking IPV seriously , asserting, for example, that cases of IPV are rarely reported in reputable newspapers unless they are sensational or end in death. Other observers (e.g., IACHR, 2012; OECD, 2014) have concurred that media presentation and coverage (or lack thereof) of this kind of violence socializes Jamaican youth to believe that IPV is normal and justified. The OECD (2010) noted that "traditional gender stereotypes are institutionalised within Jamaica's education system, the media, religion and the family” (OECD, 2010, p., 120).

\section{Institutional and Legislative Norms}

Institutionalized structures that create a climate in which violence is tolerated undoubtedly contribute to the perpetration and maintenance of gender violence (WHO, 2010, 2013). Arguably, society overtly and covertly assigns roles based on gender and socializes individuals accordingly; often laws are instituted to support those gender scripts, resulting in marginalization for those who 
deviate (OECD, 2010; OECD, 2014). Indeed, laws biased against women engender cultural conditions favourable to violence against women (Freedom House, 2015; OECD, 2014; U.S. Department of State, Bureau of Democracy, Human Rights and Labor [USDS], 2012). Freedom House (2015) underscored the continued widespread violence and discrimination against Jamaican women and highlighted the ineffectiveness of the government in protecting women and combating violence against them. For example, some observers (e.g., OECD, 2014; USDS, 2012) have pointed out that there are currently no laws addressing sexual harassment, despite the high rate of sexual harassment in the Jamaican workplace ${ }^{1}$. The OECD (2010) also noted that Jamaican legislation prohibits all discrimination based on race or religion, but not discrimination based on gender. Moreover, the OECD (2010), while acknowledging some progress toward addressing women's issues (e.g., outlawing IPV, provision for maternity leave), maintained that the laws of the country are not written in gender-neutral language and that the country's civil and penal codes still contained numerous discriminatory measures. For example, “...lesbian, bisexual and transgender women do not receive protection under the law from violence due to sexuality or gender identity and in fact are discriminated against within the criminal justice system" (OECD, 2014, p.5). The IACHR (2012) pointed out that the "shortcomings in [Jamaica's] national legislation do not deal adequately with marital rape, incest or sexual harassment, thereby encouraging impunity and leaving women without the protection of the law” (p.73). The IACHR also claimed that even when laws are amended or new ones implemented, they either do not go far enough or are still biased against women. Under the Sexual Offences Act passed in 2011, for example, marital rape is a crime only in some situations, such as:

when the parties have separated or when proceedings to dissolve the marriage or have it annulled have begun, when the husband is under a court order not to molest or cohabit with his wife, or when the husband knows he suffers from a sexually transmitted infection (USDS, 2012, p. 16).

Another telling example of a strong anti-female policy centers on women's reproductive rights. In Jamaica, under the law, a woman found guilty of terminating a pregnancy faces life imprisonment and anyone found guilty of assisting in facilitating an abortion, except to save the life of the mother, faces up to three years in prison (Amnesty International; 2014). In addition, Ustanny (2006) cited major obstacles in the judicial system for women who report sexual assault against them. In particular, she referred to the doubly traumatic experience women face because of rules that permit invasive interrogation about the sexual history of the complainant. Ustanny also called attention to the fact that women traumatized by violence against them face barriers to negotiating the systems that should be protecting them (e.g., receiving prompt medical attention). Additionally, Amnesty International (2014) pointed to the meagre clear-up rate ( 50\%) of crimes against women that are brought before the courts. In Jamaica, according to the IACHR (2012), violence against

\footnotetext{
${ }^{1}$ On December 8, 2015, a sexual harassment bill was tabled in Parliament to outlaw sexual intimidation and coercion in workplaces, institutions, and in landlord-tenant relationships.
} 
women is one of the most pervasive crimes yet the least prosecuted: violence against Jamaican women rarely results in prosecution of the perpetrator. Further, the OECD (2014) maintained that the inordinate amount of time it takes law enforcement to investigate and prosecute cases of IPV deters women from reporting crimes against them. The OECD also indicated that insufficient awareness and training among judges, prosecutors, police officers, and health professionals on violence against women is a contributing factor to Jamaica's difficulty in dealing with the problem. The IACHR (2012) asserted that the aversion of the Jamaican police to enforcing relevant IPV laws fosters victims' mistrust of the law enforcement system. Insensitivity of the criminal justice system and a tendency of courts to stereotype women in judicial decisions have also been alleged (IACHR, 2012; OECD, 2010, 2014).

\section{Consequences of IPV}

In addition to financial costs, IPV brings with it adverse health and social consequences for adults, children, and society as a whole. International research has indicated that women who experience non-lethal IPV suffer compromised physical, socioemotional, and reproductive health. When compared to women who do not report any form of IPV, women who report IPV were significantly more likely to report a greater incidence of direct injury (e.g., broken bones, cuts, burns, traumatic brain injury). They also exhibited chronic health problems such as diabetes, asthma, hypertension, cardiovascular diseases, and chronic pain syndrome (Centers for Disease Control and Prevention, U.S. Department of Health \& Human Services [CDC], 2015; Cronholm, Fogarty, Ambuel, \& Harrison, 2011). Among the RHS Jamaican sample, 30\% of IPV victims indicated that they had suffered injuries necessitating medical attention, including bruises and scratches (50\%), headaches and other pain (55\%), and more severe injuries such as cuts and burns (21\%) and stab wounds (4\%); in addition, 15\% had missed work because of the violence inflicted on them (Serbanescu et al., 2010).

The adverse psychological outcomes for IPV victims include anxiety, symptoms of posttraumatic stress disorder (PTSD), and suicidal behaviours (CDC, 2015; Cronholm et al., 2011). In one study (Houry, Kemball, Rhodes, \& Kaslow, 2006), women who reported being victims of IPV were 6 times more likely to exhibit suicidal tendencies, 3 times more likely to report symptoms of depression, and 4 times more likely to exhibit PTSD symptoms than the control group. Furthermore, victims of IPV are at increased risk of engaging in high-risk behaviours such as multiple sex partners, substance abuse, and smoking, which in turn puts them in jeopardy for further health problems (CDC, 2015). IPV undermines women's ability to exercise their reproductive rights and exacts severe costs on their sexual and reproductive health. It impedes victims' access to family planning and exposes them to pregnancy-related health risks, sexually transmitted infections, sexual dysfunction, unwanted pregnancies, miscarriages, and prenatal difficulties (CDC, 2015; Cronholm et al., 2011; Middlebrooks \& Audage, 2008). IPV puts children at risk for stunted development because it renders mothers emotionally unavailable to provide the nurturance and security that children need for optimal development (Osofsky, 2003). It also puts mothers at high risk for abusing their children (Osofsky, 2003). 
International Journal of Child, Youth and Family Studies (2016) 7(3/4): 343-363

The economic costs of IPV to society are high. IPV costs the United States, for example, an estimated 8.5 billion USD annually, excluding criminal justice services (CDC, 2015), and the economic burden is even heavier in developing countries with struggling economies. In Chile, women's loss of salary as a result of IPV amounts to about $2 \%$ of the country's Gross Domestic Product (GDP) or 1.56 billion USD (Beavers \& Kumpf, 2013). No specific IPV cost data for Jamaica could be located, but Jamaica spends about 4\% of its GDP on the direct medical cost of interpersonal violence (Ward et al., 2009). In 2006, Jamaica’s direct medical cost for interpersonal injuries accounted for $12 \%$ of the total health budget, and productivity losses due to violence-related injuries amounted to 27.5 billion JMD (Ward et al., 2009). According to Beavers and Kumpf (2013), the enactment of policies to prevent violence against women is imperative for economic growth and national development: "For most countries, it is clear that decisive action to prevent violence against women and girls will reduce state expenditures and increase productivity” (para. $6)$.

\section{Discussion and Conclusion}

The purpose of the current article is to provide an overview of the prevalence and nature of IPV against women in Jamaica and to suggest workable approaches for its prevention. IPV is a human rights transgression with serious adverse physical, sexual, socioemotional, and economic consequences, both immediate and long-term. It affects the general well-being of victims and their families, and exacts an enormous economic cost on society. Yet, IPV lacks the attention it deserves as a related but distinct issue from general societal violence. In Jamaica, research on IPV is extremely sparse; there is a large gap in the literature regarding the prevalence of IPV and its consequences for society. What is assumed about IPV in the Jamaican context has been largely extrapolated from studies done in other societies, and although those findings have been fairly consistent across cultures, generalization of research findings can be problematic. The WHO (2013) maintained that the Caribbean region is one of the regions with the least available data on IPV. Amnesty International (2014) posited that a comprehensive data collection on all forms of violence against women is needed in order to implement effective policies or laws that address women's issues. Unquestionably, extrapolation from other cultures can be helpful; however, there are unique cultural nuances that do not transmit from one culture to another. For example, it would be helpful for research to investigate not only IPV prevalence, but also the sociocultural factors that contribute to its occurrence and maintenance in Jamaica. Such research is needed since much of the available literature on IPV in Jamaica is based on anecdotal data, conjectures, and extrapolations from studies done elsewhere. It would also be helpful to understand what factors put Jamaican women at risk for IPV, the strategies women employ to cope with IPV, the particular needs of women who experience IPV, and the short- and long-term socioemotional effects on children who witness such violence. Also, it would be prudent to investigate whether Jamaican women and children identify the same consequences as women and children worldwide. To date, the sparse Jamaican IPV data come from questions about IPV that have been added on to larger studies such as the MICS and RHS. 
International Journal of Child, Youth and Family Studies (2016) 7(3/4): 343-363

Despite the limitations of some of the Jamaican data cited above, the existing data and the commensurate inferences from other sources must be taken seriously. Recently, the Jamaican government has stated its commitment to women's empowerment and to making the issue of violence against women a priority. It has also taken some positive steps in that direction (IACHR, 2012). Nonetheless, the Jamaican government has been criticized for not moving expeditiously enough to strengthen existing laws and enact new legislation to prevent and reduce the incidence of IPV and other gendered violence. According to the IACHR (2012), significant barriers to gender equality still exist and legal reform is slow. Therefore, efforts to address the Jamaican IPV problem need to be substantially increased.

Several global organizations (e.g., Amnesty International, 2014; IACHR, 2012; UNICEF, 2006a) have alluded to the role of government in the safety of its citizenry. For example, UNICEF (2006a) specifically stated that "Governments carry a primary responsibility for ensuring that children and women are safe and secure in their homes, and can take several key steps to ensure this” (p. 11). Therefore, those organizations have implored governments everywhere to move with urgency to address IPV. They have also advised that IPV is best addressed by approaches that tackle the cultural norms and systems that validate violence against women, suggesting that governments:

- embark upon a public education saturation program to raise awareness on the debilitating effects of IPV on individuals and society;

- $\quad$ enact legislation making IPV a criminal offence under all circumstances and maximizing its punishment for perpetrators;

- demand swift and judicious investigation and prosecution of violence against women;

- $\quad$ increase the allocation of social service resources to provide protection (e.g., more crisis centers and shelters) for victims; and

- $\quad$ establish and intensify training programs for key stakeholders (e.g., medical, police, justice system, clergy, public service).

Additionally, UNICEF (2006a) has called on the Jamaican private sector to recognize its moral obligation to exercise corporate social responsibility by joining the fight against IPV, highlighting the value of corporations' commitment to all employees within the workplace and recommending that corporations: (a) institute employee education and training programs to increase awareness of IPV, (b) fund and support initiatives and services for victims of IPV, and (c) persuade the government to place domestic and other gender-based violence high on the national agenda.

Male attitudes and behaviours are not the only elements that require change; female acceptance of family violence is problematic and should be addressed as well (Stop Violence Against Women, 2014). Hence, public education should extend beyond mere awareness of IPV prevalence. Campaigns should seek to empower victims and inform the public of practical ways to take action to end abuse in the family. Education also means alerting survivors to available options and resources, and apprising victims of their rights (National Resource Center on Domestic 
Violence, 2012). Furthermore, both perpetrators and victims need intensive counselling to address underlying personal deficits such as low self-image and self-efficacy, and other mental health problems. Most importantly, treatment for perpetrators should be mandated. Miller, Drake, and Nafziger (2013) reviewed the relevant IPV treatment literature and noted a variety of promising evidence-based treatment programs for IPV offenders that effectively reduced IPV recidivism. In Jamaica, treatment for alcohol use and abuse is especially critical as alcohol consumption appears to be a serious problem. When asked about their alcohol behaviours, 79\% of Jamaican men aged 20 to 24 reported a lifetime prevalence of drinking, and 41\% binge-drank in the last month. Among women in the same age group, $46 \%$ reported being drunk at some point, and $19 \%$ binge-drank in the last month (Serbanescu et al., 2010).

In addition to programs for adults, training programs for children are imperative. Helping children develop social competence, particularly constructive conflict resolution skills, has been shown to have positive developmental effects. For example, social competence has been reported to enhance emotional regulation, boost communication, improve problem solving, promote positive self-esteem, and lessen conduct problems (Smith, 2016). The assumption here is that these learned competencies will be carried forward into relationships in adulthood, thereby, lessening the prevalence of IPV.

In sum, ingrained cultural beliefs and life-long practices will not disappear with legislation and awareness alone. While prevention is the ultimate goal with regard to solving any social problem, intervention is vital for both victims and perpetrators. Considering the devastating effects of IPV on victims, families, and society, access to comprehensive services is of paramount importance. These services must include legal intervention and rehabilitation services, and these should be integrated to minimize the stress of negotiating several complex support structures. 
International Journal of Child, Youth and Family Studies (2016) 7(3/4): 343-363

\section{References}

Ackerson, L. K., Kawachi, I., Barbeau, E. M., \& Subramanian, S. V. (2008). Effects of individual and proximate educational context on intimate partner violence: A population-based study of women in India. American Journal of Public Health, 98(3), 507-514.

Amnesty International. (2014). Jamaica: Justice system must be strengthened for the effective protection of human rights: Amnesty International Submission to the UN Universal Periodic Review, May 2015 (Index No. AMR 38/0004/2014). Retrieved from https://www.amnesty.org/en/documents/amr38/0004/2014/en/

Beavers, S., \& Kumpf, B. (2013, March). Violence against women also hurts business and development. Retrieved from http://www.undp.org/content/undp/en/home/ourperspective/ourperspectivearticles/2013/03/29/ violence-against-women-also-hurts-business-and-development.html

Benson, M. L., \& Fox, G. L. (2004). When violence hits home: How economics and neighborhood play a role. Washington, DC: U.S. Department of Justice, National Institute of Justice.

Bott, S., Guedes, A., Godwin, M., \& Mendoza, J. (2012). Violence against women in Latin America and the Caribbean: A comparative analysis of population-based data from 12 countries. Washington, DC: Pan American Health Organization.

Bronfenbrenner, U. (1979). The ecology of human development. Cambridge, MA: Harvard University Press.

Campbell, J. C., Glass, N., Sharps, P., Laughon, K., \& Bloom, T. (2007). Intimate partner homicide: Review and implications of research and policy. Trauma Violence \& Abuse, 8(3), 246-269.

Capaldi, D. M., Knoble, N. B., Shortt, J., \& Kim, H. (2012). A systematic review of risk factors for intimate partner violence. Partner Abuse 3, 231-280. doi:10.1891/1946-6560.3.2.231

Centers for Disease Control and Prevention, U.S. Department of Health \& Human Services. (2015). Intimate partner violence: Consequences. Retrieved from http://www.cdc.gov/violenceprevention/intimatepartnerviolence/consequences.html

Cronholm, P., Fogarty, C., Almbuel, B., \& Harrison, S. (2011). Intimate partner violence. American Family Physician, 83, 1165-1172.

Cunningham, A., Jaffe, P. G, Baker, L., Dick, T., Malla, S., Mazaheri, N., \& Poisson, S. (1998). Theory-derived explanations of male violence against female partners: Literature update and related implications for treatment and evaluation. London, ON: London Family Court Clinic. Retrieved from http://www.lfcc.on.ca/maleviolence.pdf 
International Journal of Child, Youth and Family Studies (2016) 7(3/4): 343-363

Dutton, D. G. (2007). Female intimate partner violence and developmental trajectories of abusive females. International Journal of Men's Health, 6, 54-70.

Edwards, K. M., Mattingly, M., Dixon, K., \& Banyard, V. (2014). Community matters: Intimate partner violence among rural young adults. American Journal of Community Psychology, 53, 198-207. doi:10.1007/s10464-014-9633-7

Freedom House. (2015). Jamaica: Freedom in the world 2015. Retrieved from https://freedomhouse.org/report/freedom-world/2015/jamaica

Gass, J. D., Stein, D. J., Williams, D., \& Seedat, S. (2011). Gender differences in risk for intimate partner violence among South African adults. Journal of Interpersonal Violence, 26(14), 2764-2789. doi:10.1177/0886260510390960

Goussinsky, R., \& Yassour-Borochowitz, D. (2012). “I killed her, but I never laid a finger on her” A phenomenological difference between wife-killing and wife-battering. Aggression and Violent Behavior, 17, 553-564.

Hart, B., \& Klein, A. (2013). Practical implications of current intimate partner violence research for victim advocates and service providers (NJC 244348). National Criminal Justice Reference Service. Retrieved from https://www.ncjrs.gov/App/Publications/abstract.aspx?ID=266429

Houry, D., Kemball, R., Rhodes, K., \& Kaslow, N. J. (2006). Intimate partner violence and mental health symptoms in African American ED patients. American Journal of Emergency Medicine, 24, 444-450.

Inter-American Commission on Human Rights. (2012). Report on the situation of human rights in Jamaica (OEA/Ser.L/V/II.144 Doc.12). Retrieved from http://www.oas.org/en/iachr/docs/pdf/Jamaica2012eng.pdf

Johnson, K. B., \& Das, M. B. (2009). Spousal violence in Bangladesh as reported by men: Prevalence and risk factors. Journal of Interpersonal Violence, 24, 977-995.

Laisser, R. M., Nyström, L, Lugina, H. I., Emmelin, M. (2011). Community perceptions of intimate partner violence - a qualitative study from urban Tanzania. BMC Women's Health, 11, 13. doi:10.1186/1472-6874-11-13

Le Franc, E., Samms-Vaughan, M., Hambleton, I., Fox, K., \& Brown, D. (2008). Interpersonal violence in three Caribbean countries: Barbados, Jamaica, and Trinidad and Tobago. Pan American Journal of Public Health, 24(6), 409-421.

Meeks-Gardner, J., Powell, C., Thomas, J., \& Millard, D. (2003). Perceptions and experiences of violence among secondary school students in urban Jamaica. Pan American Journal of Public Health 14(2), 97-103. 
International Journal of Child, Youth and Family Studies (2016) 7(3/4): 343-363

Middlebrooks, J. S., \& Audage, N. C. (2008). The effects of childhood stress on health across the lifespan. Atlanta, GA: Centers for Disease Control and Prevention, National Center for Injury Prevention and Control.

Miller, M., Drake, E., \& Nafziger, M. (2013). What works to reduce recidivism by domestic violence offenders? (Document No. 13-01-1201). Olympia. WA: Washington State Institute for Public Policy.

National Child Traumatic Stress Network. (2015). Community violence. Retrieved from http://www.nctsn.org/trauma-types/community-violence

National Resource Center on Domestic Violence. (2012). Supporting public and education efforts. Retrieved from http://www.nrcdv.org/dvam/campaign-ideas

Organization for Economic Cooperation and Development. (2010). Atlas of gender development: How social norms affect gender equality in non-OECD countries. Paris, France: OECD Publishing.

Organization for Economic Cooperation and Development. (2014). Jamaica. Retrieved from http://genderindex.org/sites/default/files/datasheets/JM.pdf

Osofsky, J. (2003). Prevalence of children's exposure to domestic violence and child maltreatment: Implications for prevention and intervention. Clinical Child and Family Psychology Review, 6, 161-170.

Peek-Asa, C., Wallis, A., Harland, K., Beyer, K., Dickey, P., \& Saftlas, A. (2011). Rural disparity in domestic violence prevalence and access to resources. Journal of Women's Health, 20(11), 1743-1749. doi:10.1089/jwh.2011.2891

Planning Institute of Jamaica. (2014). Economic and social survey - Jamaica. Kingston, Jamaica: Author.

Royes, H., Samiel, S., Tate, V., \& Fox, K. (2006). Evaluation of the impact of gender-based interventions in Jamaica: Final report. Retrieved from http://198.170.76.2/jamspred/Impact_of_Gender_Based_Interventions_Jamaica.pdf

Serbanescu, F., Ruiz, A., \& Suchdev, D. B. (2010). Reproductive health survey Jamaica 2008: Final report. Kingston, Jamaica: National Family Planning Board; Statistical Institute of Jamaica; United States Agency for International Development; \& Atlanta, GA: Division of Reproductive Health, Centers for Disease Control and Prevention.

Smith, D. E. (2016). Corporal punishment of children in the Jamaican context. International Journal of Child, Youth and Family Studies, 7(1), 27-44. doi:10.18357/ijcyfs.71201615415 
International Journal of Child, Youth and Family Studies (2016) 7(3/4): 343-363

Soyibo, K., \& Lee, M. G. (2000). Domestic and school violence among high school students in Jamaica. West Indian Medical Journal, 49, 232-236.

Statistical Institute of Jamaica, \& United Nations Children’s Fund. (2013). Jamaica Multiple Indicator Cluster Survey 2011: Final report. Kingston, Jamaica: Author.

Stop Violence Against Women. (2014, October 24). Violence against women still a problem in Jamaica. Retrieved from http://www.onlyinnajamaica.com/2014/10/24/violence-againstwomen-still-a-problem-in-jamaica/

United Nations Children's Fund. (2006a). Behind closed doors. The impact of domestic violence on children. New York, NY: Author.

United Nations Children's Fund. (2006b). Violence against children in the Caribbean region: Regional assessment. Panama, Republic of Panama: UNICEF Regional Office for Latin America and the Caribbean.

United Nations Development Programme. (2012). Caribbean human development report: Human development and the shift to better citizen security. New York, NY: Author.

United Nations Office on Drugs and Crime. (2014). Global study on homicide, 2013: Trends, contexts, and data. Vienna, Austria: Author.

U.S. Department of State, Bureau of Democracy, Human Rights and Labor. (2012). Country Reports on Human Rights Practices for 2012 . Washington, DC: Author.

Ustanny, A. (2006). Against her will: A situational analysis of rape in Jamaica [Panos Caribbean Media Brief No. 11]. Retrieved from http://panoscaribbean.org/images/documents/againstherwill.pdf

Violence Policy Center. (2012). American roulette: Murder-suicide in the United States. Washington, DC: Author.

Ward, E., McCartney, T., Brown, D. W., Grant, A., Butchart, A., Taylor, M., ... Pinnock, C. (2009). Results of an exercise to estimate the costs of interpersonal violence in Jamaica. The West Indian Medical Journal, 58(5), 446-451.

Wilson, I., Graham, K., \& Taft, A. (2014). Alcohol interventions, alcohol policy and intimate partner violence: A systematic review. BMC Public Health, 14, 881. doi:10.1186/1471-2458$14-881$

World Health Organization. (2005). Summary report: WHO multi-country study on women's health and domestic violence against women. Geneva, Switzerland: Author.

World Health Organization. (2010). Preventing intimate partner and sexual violence against women: Taking action and generating evidence. Geneva, Switzerland: Author. 
International Journal of Child, Youth and Family Studies (2016) 7(3/4): 343-363

World Health Organization. (2013). Global and regional estimates of violence against women: Prevalence and health effects of intimate partner violence and non-partner violence. Geneva, Switzerland: Author.

World Health Organization. (2016). Violence against women [Fact sheet No. 239]. Retrieved from http://www.who.int/mediacentre/factsheets/fs239/en/

Zakar, R., Zakar, M. Z., \& Abbas, S. (2016). Domestic violence against rural women in Pakistan: An issue of health and human rights. Journal of Family Violence, 31(1), 15-25. 